\title{
DiOTtos
}

Revista de Comunicación Digital

\section{La conversación digital en torno al hashtag \#RespetoAIDolorDeMadre en Panamá}

\author{
The digital conversation around \\ \#RespetoAIDolorDeMadre in Panamá
}

\section{Marina Acosta}

macosta@sociales.uba.ar

Universidad de Buenos Aires

Universidad Nacional Arturo Jauretche

\author{
Claire Nevache \\ cnevache@cieps.org.pa \\ Centro Internacional de Estudios \\ Políticos y Sociales
}

\section{Resumen}

En Panamá, al igual que en otros países de la región, los grupos conservadores religiosos han conseguido convertirse en verdaderos actores políticos, impidiendo cualquier avance en políticas públicas o normativas relativas a la sexualidad y el género. En este contexto, fue presentado un proyecto de ley "que da identidad a los bebés fallecidos en el vientre materno" propuesto por una diputada de la mayoría parlamentaria, vocera notoria de los grupos conservadores anteriormente mencionados. En las redes sociales se activó la polémica que, a su vez, se polarizó entre cuentas usuarias de la etiqueta \#TuNombreMiRecuerdo, a favor del proyecto y \#RespetoAIDolorDeMadre, en contra. En este último hashtag, impulsado por activistas feministas, cientos de mujeres compartieron experiencias de sufrimiento por la pérdida de embarazos. El objetivo general de la investigación es analizar la conversación digital en torno al \#RespetoAIDolorDeMadre. Desde el punto de vista metodológico, recurrimos al social media mining, realizado con diferentes herramientas de asistencia que se utilizan para extraer y organizar información que no es posible obtener mediante métodos estadísticos convencionales. El estudio se enmarca en los desarrollos teóricos que postulan al espacio digital como una nueva vía de participación donde los actores sociales, también, pueden debatir problemáticas que los afectan. Dentro de los principales hallazgos destacan que la conversación digital registra comunidades agrupadas con conexiones sólidas y conversaciones densas, que las cuentas más retuiteadas pertenecen a activistas feministas y que las cuentas más mencionadas por los/las usuarios/as en sus interacciones corresponden al sistema político.

\section{Palabras clave}

ciberactivismo, feminismo, aborto, Panamá

Recibido: 09/12/2019

Aceptado: 19/03/2020

Cómo citar este artículo:

Acosta, M. y Nevache, C. (2020). La conversación digital en torno al hashtag \#RespetoAIDolorDeMadre en

Panamá. Dígitos. Revista de Comunicación Digital, 6: 13-30. DOI: 10.7203/rd.v1i6.171 


\begin{abstract}
In Panama, as in other countries in Latin America, conservative religious groups have managed to become true political actors, preventing any progress in public or regulatory policies related to sexuality and gender. In this context, a bill « that gives identity to deceased babies in the womb » was proposed by a representative of the parliamentary majority, a notorious spokeswoñqn of the mentioned conservative groups. In the social networks the controversy was activated that, in turn, was polarized between user accounts of the \#TuNombreMiRecuerdo label, in favor of the project and \#RespetoAIDolorDeMadre, against. In the second hashtag, launched by feminist activists, hundreds of women shared experiences of suffering from pregnancy loss. The main objective of this research is to describe the digital conversation around the \#RespetoAIDolorDeMadre. The research uses the social media mining, carried out with different assistance software used to extract and organize information otherwise impossible to obtain using conventional statistical methods. The study framework comes from the theoretical developments of cyberactivism. The main findings are that the digital conversation registers communities with solid connections and dense conversations, that the most retweeted accounts belong to feminist activists and that the accounts most mentioned by users in their interactions correspond to the political system.
\end{abstract}

\title{
Keywords
}

cyberactivism, feminism, abortion, Panama. \#RespetoAlDolorDeMadre en Panamá

\section{Introducción}

El 15 de julio de 2019, apenas dos semanas después de la instalación de la electa Asamblea Nacional de Panamá, se presentó el anteproyecto de ley 029 . Luego se convertiría en el proyecto de ley 018 , bajo el título "De identidad para bebés fallecidos en el vientre materno", siguiendo otros proyectos legislativos muy similares en la región. El anteproyecto fue presentado por la diputada Corina Cano y respaldado por diputados del partido Movimiento Liberal Republicano Nacionalista (MOLIRENA), parte de la alianza mayoritaria con el Partido Revolucionario Democrático (PRD).

El proyecto de ley 018 prevé la creación de un nuevo libro en el registro civil (Libro de Defunciones de Personas Concebidas no Nacidas) donde se inscribirían en un plazo de 72 horas todas las defunciones que ocurrieran en el vientre materno cualquiera sea la causa de la muerte, edad gestacional o peso que tuviera al momento del fallecimiento. La inscripción requeriría un certificado médico y daría lugar, a su vez, a la expedición de un certificado de fallecimiento que permitiría la inhumación.

Rápidamente, en las redes sociales, se activó la polémica. El ciberdebate se polarizó entre cuentas usuarias de la etiqueta (importada de otros países) \#TuNombreMiRecuerdo, a favor del proyecto y \#RespetoAIDolorDeMadre, en contra. Este último hashtag,

1. La diputada Cano es conocida por su calidad de vocera de la Alianza Panameña por la Vida y la Familia, organización movilizada en contra de la llamada "ideología de género" y de las políticas públicas relacionadas con temas relativos al género y la sexualidad. 
impulsado por activistas feministas, fue creado para visibilizar sensibilidades; por eso, en él, cientos de mujeres además de posicionarse frente al tema compartieron experiencias de sufrimiento por la pérdida de embarazos. Twitter ${ }^{2}$ se convirtió entonces en escenario de la indignación, como había ocurrido en otros casos (Acosta, 2018b; Acosta \& Lassi, 2019; Clark, 2016; Larrondo et al., 2019; Laudano, 2017).

La literatura ha focalizado en las formas de comunicación que utilizan las protestas sociales en red (Alcazan et al., 2012; Castells, 2012; Cruells e Ibarra, 2013; Gerbaudo, 2012; 2016; Sádaba, 2012; Sorj y Fausto, 2016; Treré y Barranquero, 2013). El avance del feminismo ha llevado también a indagar en las posibilidades que ofrecen las plataformas digitales para el movimiento (Acosta, 2018a; 2018b; Arias Rodríguez, 2017; Cook y Hasmath, 2014; Fotopoulou, 2014; Gago, 2019; Molpeceres y Filardo, 2020; Núñez Puente y Fernández Romero, 2017; Sádaba y Barranquero, 2019; Tarullo y García, 2020). Empero, para el caso panameño, no se encuentran trabajos que hayan reparado específicamente en el ciberactivismo feminista o el "feminismo del hashtag" (Caro, 2015; Loza, 2014). El único antecedente que encontramos se concentra en la iniciativa ciudadana de 2019 en contra de la reelección de funcionarios públicos que tuvo también su capítulo en las redes sociales bajo la etiqueta \#NoALaRelección (Brown Araúz, 2020).

Así, en esta investigación buscamos analizar la conversación digital en torno al hashtag \#RespetoAIDolorDeMadre y las interacciones entre usuarios/usuarias que se dieron a su interior. Estimamos importante, en este sentido, aportar análisis empírico al campo de estudios del ciberactivismo feminista en Panamá dado que no encontramos trabajos que hayan reparado, stricto sensu, en él.

\section{Contexto}

\subsection{El proyecto de ley 018}

El proyecto de ley 018 está antecedido de una exposición de motivos que explica las razones por las que los proponentes consideran necesaria esta legislación. Su principal objetivo sería la atención adecuada del duelo perinatal a través de un reconocimiento social de la pérdida de embarazo ${ }^{3}$. No obstante, el contexto regional latinoamericano está sacudido por el debate sobre la despenalización o la legalización de la interrupción voluntaria del embarazo. Las discusiones en torno a este tema en los congresos argentinos, ecuatorianos, bolivianos y chilenos desde el 2017, así como las movilizaciones simbolizadas por los pañuelos verdes por un lado y los pañuelos celestes por el otro, han colocado el tema en el centro de la política latinoamericana.

En este marco, al retomar argumentos muy similares a los utilizados en contra de la despenalización o legalización del aborto, el proyecto de ley se enmarcó en otro debate, más allá del duelo perinatal. Es el caso, por ejemplo, de las referencias en la exposición de motivos al derecho a la identidad y a la vida del "bebé por nacer" y las digresiones

2. Luego de Facebook, Instagram y LinkedIn, Twitter es la cuarta plataforma digital con más usuarios/as en Panamá (322.5 mil de usuarios/as), lo que equivale al 15\% de la cantidad de usuarios/as activos/as en redes sociales. El informe Latinobarómetro 2018 señala que el 15.3\% de panameños y panameñas son usuarios/as de esta red social que, además, ocupa el puesto 15 de los websites más visitados (Hootsuite We Are Social, 2019).

3. El anteproyecto puede consultarse en: https://www.asamblea.gob.pa/APPS/SEG_LEGIS/PDF_SEG/PDF SEG 2010/PDF_SEG_2019/2019 P 018.pdf 
sobre los derechos humanos desde la concepción. Asimismo, en su artículo 10 prevé el establecimiento del 25 de marzo, día de la Anunciación, como Día del Niño por Nacer, tal como ha sido el caso en otros países, impulsado por movimientos autodenominados "provida" (Vaticano, 1999; Aciprensa, sin fecha). Finalmente, la militancia política en grupos conservadores de la principal proponente del proyecto terminaba de alertar a los grupos feministas sobre las posibles intenciones escondidas detrás de él.

La Comisión de la Mujer, la Niñez, la Juventud y la Familia de la Asamblea Nacional se reunió, en primer debate, el 22 de agosto de 2019. En esa ocasión, se pronunciaron a favor del proyecto varias organizaciones tales como la Asociación Cristiana de Comunicación (directamente ligada a la mayor megaiglesia evangélica del país, la Comunidad Apostólica Hossana), un psicólogo que se presentó a título individual, el coordinador de la Unión de Asociaciones y Padres de los Centros Educativos Particulares de Panamá (cercano a la Alianza Panameña por la Vida y la Familia), la magistrada Judith Cossú del Tribunal Superior de la Niñez y Adolescencia, así como dos mujeres que habían pasado por un duelo perinatal. Las organizaciones a favor de la ley sustentaron sus intervenciones con argumentos más bien emocionales relacionados con la pérdida de un embarazo y el proceso de duelo (Alianza Panameña por la vida y la familia, 2019).

En contra del proyecto de ley se pronunciaron veintidós organizaciones de la sociedad civil -la mayoría organizaciones de mujeres- con argumentos científicos (los términos del proyecto de ley no se adecuaban a las definiciones técnicas médicas y no sustentaba cómo la inscripción facilitaría el proceso de duelo), administrativos (la duplicación de los procedimientos con protocolos ya establecidos) y legales (la problemática de la creación de una nueva figura de persona en el ordenamiento jurídico).

Entre 2016 y 2019, las organizaciones religiosas y conservadoras ganaron varias batallas jurídicas y políticas; específicamente, contra un proyecto de ley que establecía la educación sexual en las escuelas (2016), para la no-creación de una dirección de igualdad de género y desarrollo en el Ministerio de Educación (2016), la retirada de manuales escolares que mencionaban la problemática de la discriminación contra parejas del mismo sexo (2017), la no aprobación de la ley marco para la creación del Sistema de Garantías y Protección Integral de Niñez y Adolescencia (2018) y la inmovilidad de la Corte Suprema de Justicia sobre las demandas en inconstitucionalidad relativa al matrimonio entre personas del mismo sexo desde 2016 (Nevache, 2017; 2018).

Szwako y Sivori (2019) han mostrado que la gramática familista, entendida por ellos como un recurso discursivo que hace referencia constante a la familia y que comprende una ambigüedad entre un ideal igualitario (los derechos humanos) y la jerarquía propia de las familias, era un discurso utilizado habitualmente para difundir pánico moral. En rigor, hallaron que tal recurso había sido utilizado por distintas fuerzas políticas con efectos claves en episodios recientes de la política latinoamericana. Así, en el contexto del debate relativo al proyecto de ley 018 , algunas personas dentro del movimiento feminista decidieron dejar de lado momentáneamente los argumentos jurídicos y científicos que solían emplear para apropiarse de este recurso discursivo, más propio de los grupos conservadores.

En Panamá, los datos de la Encuesta CIEPS de Ciudadanía y Derechos muestran que, a pesar de una muy baja confianza interpersonal y en las instituciones, la familia es la que capitaliza el mayor grado de confianza entre la población con $57.8 \%$ de las personas que responden confiar bastante o mucho en sus familiares (Encuesta CIEPS de Ciudadanía 
y Derechos en prensa). Aun así, la utilización de este vocabulario familista no estuvo exento de debate y de dilemas dentro del movimiento feminista en tanto significaba reapropiarse del rol reproductivo que ha sido desde siempre una parte medular del sistema de género (Palomar Verea, 2004).

\subsection{El movimiento feminista panameño}

El feminismo panameño tiene raíces antiguas, con un partido feminista fundado en 1923 y campañas para el voto femenino desde el inicio de los años veinte. Sin embargo, hasta hace unos años existía cierto "estancamiento" y envejecimiento del movimiento. Por ejemplo, con una tasa de mujeres inferior al 20\% en la Asamblea Nacional, Panamá es uno de los últimos países de la región sin ley de cuotas electorales vinculante (Brown Araúz y Freidenberg, 2019) y las tentativas para prohibir la violencia mediática no han podido ser reglamentadas (Miller, 2016).

La investigación académica sobre el feminismo panameño se ha centrado en los primeros momentos del movimiento hasta los años ochenta (Aparicio et al., 2002; Bonilla, 2007; Marco, 1997a; 1997b; 2002; 2005; 2007; 2010; 2018; Ungo, 2000) aunque también comenzaron a investigarse casos paradigmáticos que tuvieron a las mujeres panameñas liderando movimientos reivindicativos más recientes (Arce, 2008; Moreno de Cuvillier, 2017).

\section{Marco Teórico}

Para el activismo, el espacio digital se presenta como una nueva vía de participación donde se pueden debatir las distintas problemáticas que afectan a los sistemas sociales. En efecto, el uso de la tecnología con fines políticos permitió el surgimiento de movimientos en línea: ecologistas, pacifistas, religiosos, sindicales, feministas y defensores de derechos civiles (Cohen \& Rai, 2000). Es que los actores sociales recurren a las posibilidades que ofrecen las nuevas tecnologías de la comunicación para amplificar las bases de sustento de su protesta (Cammaerts, 2012; Cammaerts et al., 2013; Casero-Ripollés, 2015; Castells, 2009; 2012; Welp, 2015).

Se trata de un nuevo escenario que deja atrás el momento reactivo frente a las plataformas digitales para ingresar a un período propositivo que las ve como lugares donde también puede desarrollarse la actividad política (Cammaerts et. al, 2013; Sádaba, 2012). En sintonía con esa idea, la apropiación que los/las usuarios/as hacen de las tecnologías de la información y la comunicación las convierte en tecnologías del aprendizaje y el conocimiento al tiempo que proporcionan empoderamiento (Reig, 2012).

Por su naturaleza, las redes digitales siempre están abiertas y constituyen una nueva vía de participación ciudadana que, a su vez, cobra un nuevo valor en términos de activismo y deliberación sobre los asuntos públicos (del Campo García y Resina de la Fuente, 2010; Sampedro y Resina de la Fuente, 2010; Resina de la Fuente, 2010).

Las tecnologías digitales de la autocomunicación de masas (Castells, 2009) proporcionan a la acción colectiva un poderoso canal que permite, por un lado, la difusión y persuasión de sus acciones y, por otro, la conformación de su identidad pública y colectiva (Diez y Laraña, 2017: 109). De allí que las organizaciones cuyo propósito sea generar cambios de actitudes y comportamientos vean a las plataformas digitales como espacios de comunicación (Sádaba y Barranquero, 2019). 


\subsection{Conversación digital}

Las redes sociales ofrecen espacios para que los/las usuarios/as conversen, de manera virtual, con otros/otras usuarios/as. Empero, lo que los usuarios expresan en ella no constituye una muestra representativa de la población.

Al constituirse como una red de microblogging, Twitter permite abrir debates y crear comunidades de usuarios/as con ideas (más o menos) parecidas (Arroyas-Langa et al., 2018; Larrondo, 2019). La disputa por la interpretación encuentra en las etiquetas su eje de articulación. Los hashtags, o etiquetas son creados por los propios/as usuarios/as y permite agrupar contenidos. Poseen una dimensión performativa; es decir, "hablan por sí mismos, para sí y para otros con la finalidad de extender el acontecimiento entre los ecos de la retroalimentación mediática global" (Toret, 2013: 62). Las etiquetas, en efecto, deben ser fáciles de memorizar para fomentar la viralidad y la difusión entre usuarios/as (Castelló, 2013).

Definimos, entonces, a la conversación digital que se genera en las redes sociales como las interacciones dialógicas (tuits, retuits, favoritos, respuestas, menciones) que se activan alrededor de los hashtags (Larrondo et al., 2019: 209).

\subsubsection{Comunidades digitales}

En las plataformas digitales suele operar el principio de homofilia que, grosso modo, postula que los individuos con características similares son más propensos a asociarse entre sí; al compartir creencias, ideas y valores, la comunicación y las relaciones entre ellos se da de manera más fácil (Lazarsfeld \& Merton, 1954).

Los/las usuarios/as que se vinculan en las plataformas digitales pueden discutir, apoyarse, coincidir o entrar en desacuerdo. Las comunidades virtuales son un conjunto de usuarios/as cuyos miembros interactúan entre sí e independientemente de sus posturas frente a los temas activan una relación; las une la transacción, el interés o las vinculaciones personales (Gupta \& Kim, 2004).

En Twitter, las comunidades se clasifican, de acuerdo con la forma que toman, en los siguientes arquetipos (Smith, et al., 2014: 3-5):

1) grupos polarizados: se caracteriza por dos grandes y densos grupos que poseen poca conexión entre ellos. Discuten tópicos que, generalmente, producen alta controversia política aunque no esgrimen demasiados argumentos y se ignoran mutuamente;

2) grupos densos: en ellos se registra una alta interconexión de sujetos con muy pocos participantes aislados lo que genera una conversación densa;

3) clusters de marca: se trata de agrupaciones de baja intensidad (con poca interconexión) que discuten acerca de marcas, productos o servicios o sujetos de reconocimiento público como celebridades;

4) comunidades agrupadas: algunos tópicos populares pueden desarrollar muchos grupos pequeños que se forman alrededor de nodos con sus propias audiencias, influencers y fuentes informativas;

5) redes de difusión (broadcast): en ellas los usuarios/as siguen, difunden y comentan la actualidad pero no establecen, necesariamente, conversaciones con otros nodos. El centro de la red suele ser un medio de comunicación tradicional y reconocido;

6) redes de apoyo o soporte: en rigor, no se trata de una conversación sino de quejas de clientes de empresas o servicios que forma una estructura diferente del patrón de la red de difusión. 


\section{Metodología}

El objetivo general de este trabajo es analizar la conversación digital, en Twitter, en torno a la etiqueta \#RespetoAIDolorDeMadre. En tanto, son objetivos específicos: 1) precisar cuáles fueron las cuentas más retuiteadas; 2 ) identificar las cuentas más arrobadas por los/las usuarios/as; 3) dar cuenta de la formación de las comunidades virtuales.

La propuesta empírica de esta investigación es exploratoria y descriptiva (Dankhe, 1986). Como las plataformas digitales son fuente de una enorme cantidad de datos que se producen de manera masiva (big data), recurrimos al social media mining en tanto nos permite capturar, representar, analizar y visualizar los datos generados por los/ las usuarios/as para descubrir ciertos patrones tales como la influencia y la viralización (Zafarani et al., 2014)4. El análisis de las interacciones sociales permite, por un lado, conocer el tipo de estructura de la red social que se genera en torno a un hashtag y, por otro, utilizar la estructura de la red para establecer métricas que determinan la influencia y viralización de los/las usuarios/as que participaron de dicha red (Jiménez, 2016).

El diseño de la estrategia metodológica ha sido dividido en tres etapas: 1) captura de datos; 2) análisis de datos con open softwares informáticos; 3) visualización de los datos.

Para capturar los datos recurrimos a la herramienta de análisis de hashtags, Tweet Binder, que nos proporcionó un dataset (conjunto de datos), con todas las publicaciones del período, que a su vez convertimos en un archivo ".csv" (comma-separated values) para poder importarlo desde las herramientas que utilizamos para el análisis de los datos.

Los datos fueron manipulados, por un lado, en RStudio y, por otro, en Gephi. Con la primera herramienta generamos un dataframe $e^{5}$ (con variables categóricas y numéricas) que nos permitió conocer cuáles fueron las cuentas más activas, cuáles las más retuiteadas y cuáles las más mencionadas. Para acceder a ciertos aspectos técnicos de la red estudiada, la segunda herramienta nos permitió identificar las características (modularidad, cantidad de aristas y módulos) de los nodos construidos en las diferentes interacciones de los/las usuarios/as. Las relaciones entre nodos (usuarios) y aristas (relación direccional entre nodos) son esenciales para construir datos sobre la articulación, densidad, organicidad y conectividad de una determinada red (Ábrego et al., 2018).

La visualización de los datos la hicimos con RStudio, Tableau y Gephi que, puntualmente, nos ayudó a presentar el grafo (conjunto de nodos y aristas) de la interacción de los/las usuarios/as que resultó de la aplicación del algoritmo ForceAtlas 2. En nuestro análisis, como hemos dicho, los nodos se enlazan (aristas) con otros nodos y constituyen la estructura de la red (Giraldo-Luque et al., 2018).

Dejamos en claro que somos conscientes de que cuando se realizan estudios sobre hashtags operan diferentes sesgos. En nuestro caso, el principal sesgo es que sólo estamos analizando las interacciones que la Application Programming Interface (API) de Twitter decide mostrar; además de que en esta investigación no han sido tenidos en cuenta lo que los/las usuarios/as dicen en sus publicaciones.

4. Algunos trabajos han utilizado una metodología similat para describir fenómenos de participación feminista en Twitter (Acosta y Lassi, 2019; Acosta y Lassi, 2020; Larrondo, 2019; Orbegozo et al., 2019; Santini et al., 2017).

5. Clase de objeto en el lenguaje de programación $R$. 


\subsection{Corpus de análisis}

El análisis se aplicó a un corpus de 6.716 tuits publicados por 1.639 usuarios/as únicos del hashtag \#RespetoAIDolorDeMadre. Las unidades de análisis fueron los tuits individuales. El universo analizado comprende los tuits y retuits publicados entre el 7 de septiembre y el 6 de octubre de 2019, período que coincide con el mes posterior al lanzamiento de la etiqueta promovida por las feministas panameñas.

Una vez que con RStudio obtuvimos las cuentas con mayor actividad, observamos directamente esos perfiles de Twitter y las reacciones generadas por sus publicaciones. Los/las usuarios/as más activos/as en una etiqueta nos ayudan a detectar su influencia en el tema. En tanto la popularidad o alcance de una publicación nos permite medir las interacciones que produce (respuestas, favoritos y en especial retuits) para así dar cuenta de la "conversación" digital.

Los tuits fueron clasificados en función de las entidades encontradas; esto es, imágenes, hashtags o menciones a otros usuarios, siguiendo la propuesta de Congosto (2015). El retuit (RT) es un mecanismo que se utiliza para retransmitir tuits de otros/otras usuarios/ as, de manera que el mensaje aparezca multiplicado y asociado al autor/a original; en general, dar retuit a un/una usuario/a implica apoyar lo que éste/ésta publica.

\section{Resultados}

Durante el período estudiado se recolectaron 6.716 tuits de la actividad registrada de 1.639 usuarios/as únicos/as en el \#RespetoAlDolorDeMadre, con un promedio de 4.09 tuits por cuenta y un total de 5.711 retuits.

El estudio de las dinámicas de la discusión digital y de los picos de conversación en torno al \#RespetoAIDolorDeMadre permite identificar varias etapas con diferentes actores y factores de expansión del debate. En un primer momento, la discusión fue lanzada por activistas feministas, con un eco más bien moderado (del 7 al 10 de septiembre). En un segundo momento (11 y 12 de septiembre), la actividad de las feministas tuvo un eco (relativamente) fuerte en distintas personalidades e influencers. En un tercer momento, la discusión encontró una caja de resonancia en los medios de comunicación tradicionales y en las repercusiones del comunicado de la Conferencia Episcopal apoyando el proyecto (12 de septiembre):

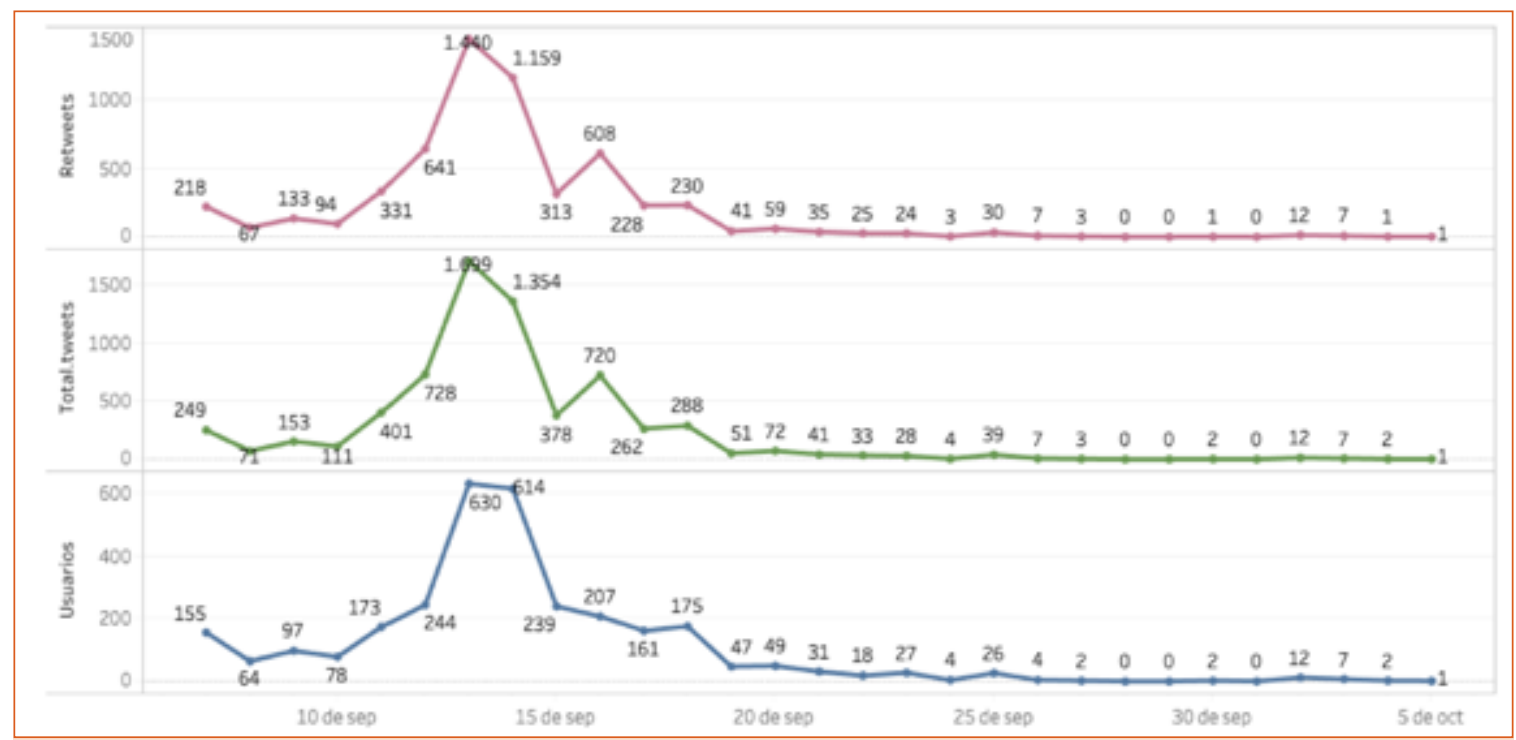

Figura 1. Actividad de la etiqueta. | Fuente: Elaboración propia. 
Como puede verse en la Figura 1, la mayor parte de la actividad en torno al \#RespetoAlDolorDeMadre tuvo lugar entre el 10 de septiembre y el 19 de septiembre, período en el que la Comisión de la Mujer había anunciado que retomaría el debate en torno al proyecto de ley. En ese lapso se contabilizan 5.854 tuits con 5.044 RT; en otras palabras, el $87.16 \%$ de los tuits totales y el $88.32 \%$ de los RT se concentró allí.

El impacto durante estos días trascendió las redes sociales. Después de que la etiqueta se convirtiera en trending topic, varios medios de comunicación tradicionales publicaron notas sobre el tema (Carrasquilla Reina, 2019; Redacción de TVN Noticias, 2019). El 12 de septiembre, la Conferencia Episcopal emitió un comunicado ${ }^{6}$ apoyando el proyecto de ley, en reacción al clima de opinión negativo que se había generado en la conversación digital. El 13 de septiembre registró la mayor cantidad de tuits, de RT y usuarios/as que participaron del hashtag. Es decir, el 21.44\% de los tuits totales se concentraron el día posterior al comunicado de la Conferencia Episcopal.

\subsection{Usuarios/Usuarias}

Dentro del universo de usuarios/as que registraron actividad en el hashtag \#RespetoAIDolorDeMadre seguimos en particular las diez cuentas con más RT:

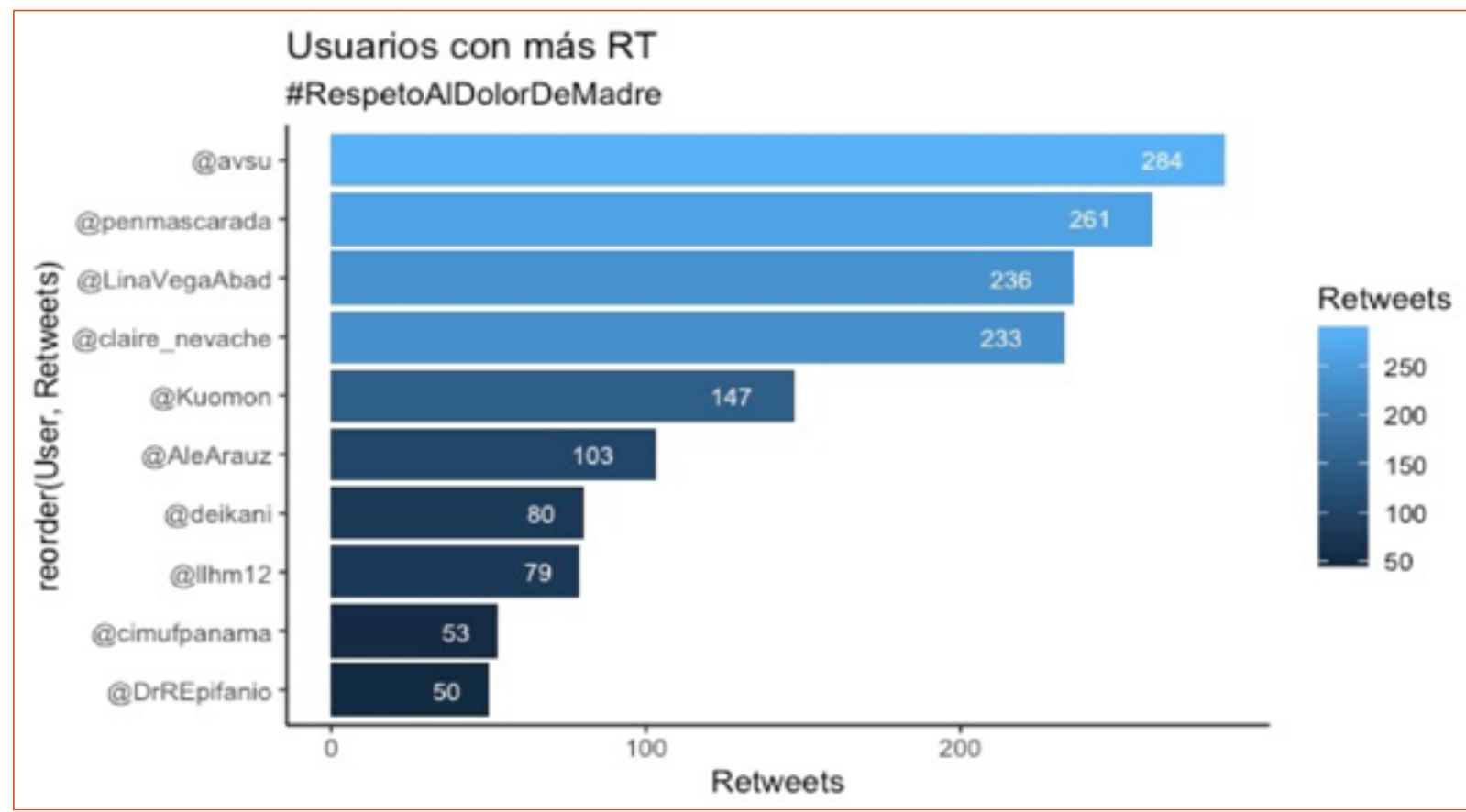

Figura 2. Cuentas con más retuits. | Fuente: Elaboración propia.

La cuenta que recibió más retuits (284) fue @avsu, de Ana Victoria Sánchez Urrutia, constitucionalista y experta en bioética, miembro del comité asesor de la Organización Mundial de la Salud sobre edición genómica. Le siguen @penmascarada, una cuenta fake que se presenta como Katrina empollerada (261 retuits); @LinaVegaAbad, constitucionalista y reconocida periodista (236 retuits); @claire_nevache, politóloga asociada al Centro Internacional de Estudios Políticos y Sociales (CIEPS Panamá) (233 retuits); @Kuomon, de Alfredo Berguido, abogado (147 retuits); @AleArauz, de Alejandra Araúz, actriz y activista feminista (103 retuits); @deikani, de Deika Nieto, abogada y defensora de los derechos humanos (80 retuits); @llhm12, estudiante de derecho (79 retuits); @cimufpanama, de la Coalición Internacional de Mujeres y Familias (CIMUF) (53 retuits) y @DrREpifanio, del obstetra Enrique Epifanio (50 retuits). 
Vale decir que entre las diez cuentas más retuiteadas encontramos siete con perfiles académicos y/o activistas, una organización feminista, un personal de la salud y una cuenta paródica o fake.

\subsection{Cuentas mencionadas}

Durante la conversación, los/las usuarios/as arrobaron a ciertas cuentas. Nos hemos concentrado en los/las cinco usuarios/as más mencionados/as en las publicaciones que componen el corpus:

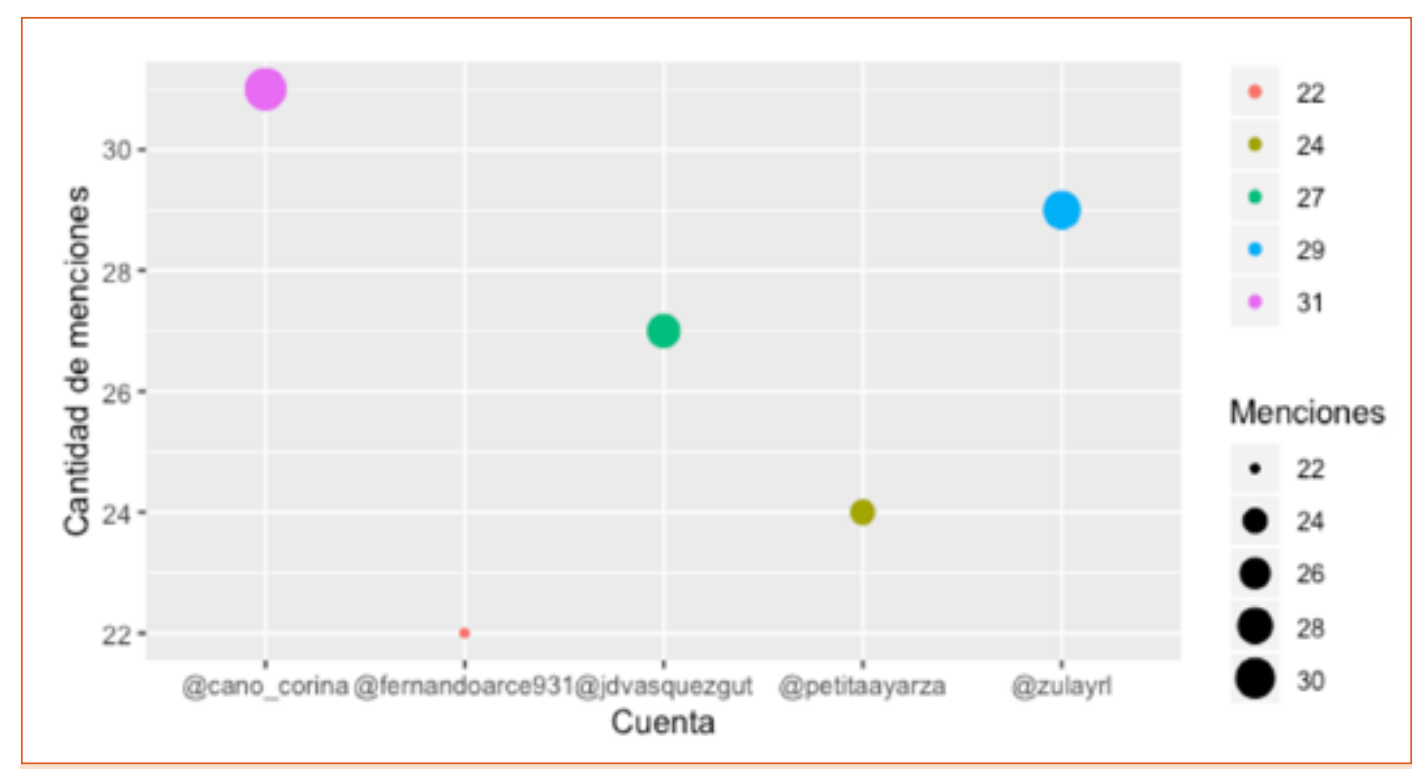

Figura 3. Cuentas con más menciones Fuente: Elaboración propia.

Como se advierte en la Figura 3, las cuentas que fueron más mencionadas por los/ las usuarios/as refieren a las diputadas y diputados que conforman la Comisión de la Mujer, la Niñez, la Juventud y la Familia, donde se debatía el proyecto de ley. La presión de los/las usuarios/as estuvo centrada allí.

La cuenta con más menciones (31 menciones) fue la de la diputada Corina Cano del MOLIRENA (@cano_corina), proponente del proyecto de ley. Le sigue la de la diputada Zulay Ródríguez (@ZulayRL), del partido PRD (29 menciones) que tiene la particularidad de ser vicepresidenta de la Asamblea Nacional y presidenta de la Comisión de la Mujer. El tercero con más menciones es el diputado independiente Juan Diego Vásquez (@ JDVasquezGut), que fue el diputado más joven así como el más votado de las últimas elecciones (27 menciones), seguido por las de la diputada Petita Ayarza (@PetitaAyarza) del PRD (24 menciones) y del diputado Fernando Arce (@fernandoarce931) del MOLIRENA (22 menciones). Las cinco cuentas con más menciones pertenecen todas a las de diputados y diputadas miembros de la Comisión de la Mujer.

\subsection{Comunidades}

El análisis de comunidades revela datos que aportan información adicional. La estructura del grafo, que presentamos a continuación, emerge de las relaciones (seguidores y RT) entre cuentas. Gephi detectó un total de 1.586 nodos, 2.532 aristas y 11 comunidades en la conversación digital en torno al hashtag \#RespetoAIDolorDeMadre. A través de la combinación de los algoritmos Force Atlas 2 y Noverlap graficamos la interacción entre usuarios/as y obtuvimos las imágenes nodales: 


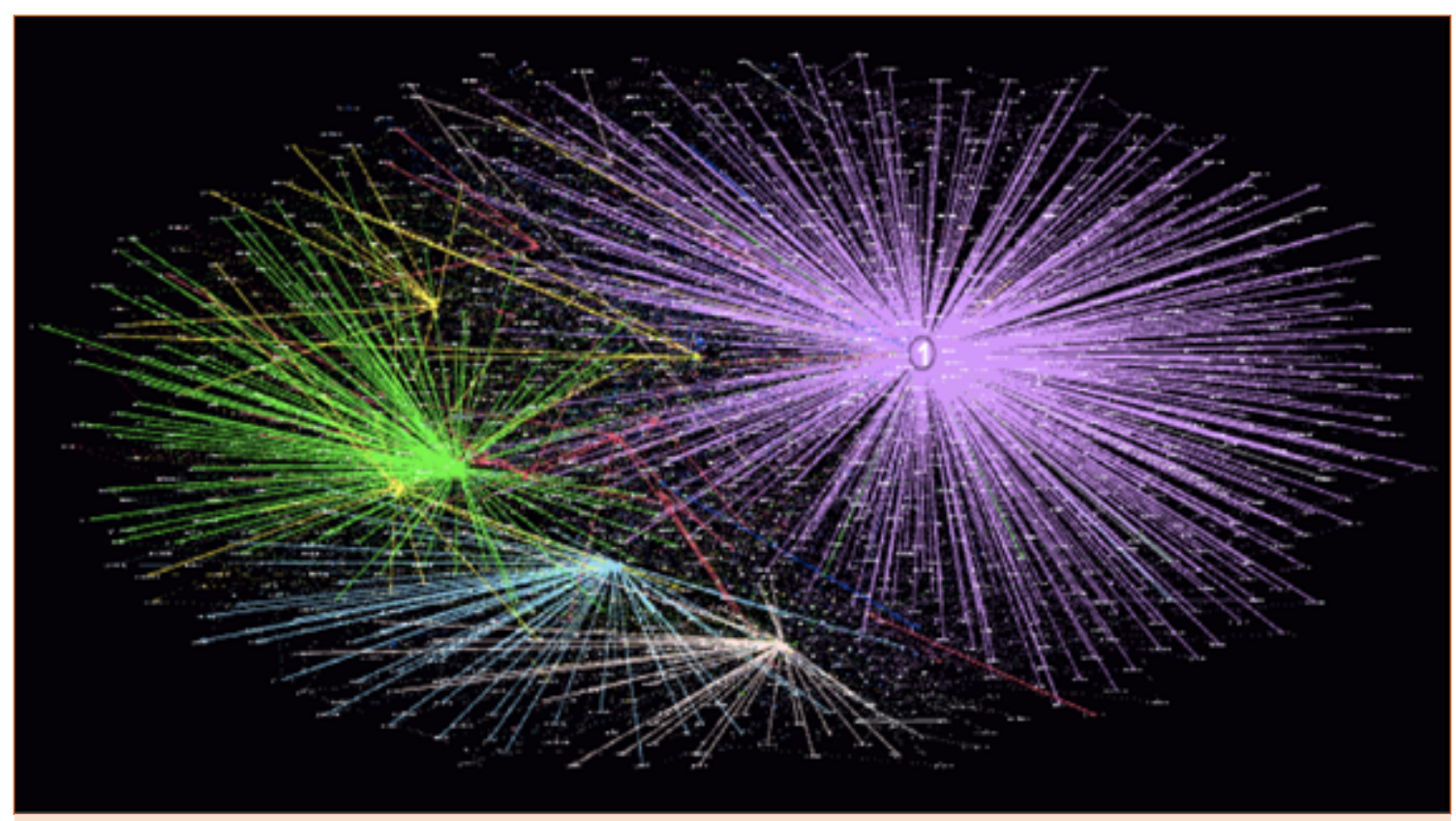

Figura 4. Grafo. | Fuente: Elaboración propia.

Uno de los elementos clave para analizar una red es la modularidad que expresa su división en módulos (comunidades). El grafo posee un grado de modularidad total de 0.47; se trata de un buen valor dado que las comunidades encontradas están interna y densamente conectadas.

Los colores de la Figura 4 nos aportan datos de las comunidades con mayor modularidad. Como se ve, hay una comunidad destacada -en color morado y con el número 1- que representa el $47.87 \%$ de partición de la red. Los nodos están conectados, fuertemente, entre sí. Esto demuestra el rol central que tuvo este cluster dentro de la conversación.

En la comunidad morada podemos encontrar varias de las figuras movilizadas en contra de la corrupción y la reelección en la campaña electoral de 2019, que en ese entonces se mostraron muy participativas en Twitter. Este movimiento que tuiteó de forma masiva bajo la etiqueta \#NoALaRelección estaba dirigido de forma específica a la Asamblea Nacional que era, después de los partidos políticos, la institución que generaba mayor desconfianza entre la población (61.83\% de la población según los datos del Barómetro de las Américas, 2018/2019). Varias figuras del Movimiento Independiente (MOVIN), programas de radio (Sal y Pimienta, Pauta en radio) e influencers participaron de esa causa. Ocho de las cuentas más activas en la etiqueta \#NoALaRelección también tuitearon bajo la etiqueta \#RespetoAIDolorDeMadre; siete de ellas se encuentran en esta comunidad. Por lo tanto, podemos suponer que la discusión digital en contra del proyecto de ley, al menos en esta comunidad, se nutrió de un sentimiento de hostilidad hacia la Asamblea Nacional, en general, y a la diputada Cano, en particular, proponente del proyecto y cuya cuenta fue la más arrobada en la conversación digital, como hemos visto. Al aplicar la métrica de centralidad de vector, comprobamos que en esta comunidad hay un alto número de nodos interactuando. Notamos, no obstante, que hay pocas vinculaciones con otros módulos importantes, y con gran impacto, representados con los colores verde, celeste, blanco y rosado.

La comunidad celeste está relacionada con el mundo cultural y el activismo feminista y LGBT. En este grupo se encuentran la mayoría de las cuentas institucionales de organizaciones feministas; por caso, Espacio Encuentro de Mujeres y Encuentro Intergeneracional Feminista. Su enfoque está sustentado más bien en el derecho de las mujeres a disponer, en libertad, de su cuerpo. 
Los módulos verde y blanco están formados por cuentas sensibilizadas con la problemática feminista pero sin pertenencia a alguna organización. Se trata de usuarios/ as que se expresan a título personal.

La comunidad rosada, que podríamos describir como de enlace entre los clusters, se caracteriza por la fuerte presencia de medios de comunicación tradicionales y de periodistas, otros actores sociales tales como organizaciones no gubernamentales de derechos humanos (el Centro de Iniciativas Democráticas y los Independientes pro Derechos Humanos) y líderes religiosos opuestos al proyecto (el rabino Gustavo Kraselnik). El rol de este módulo confirma que los medios de comunicación, así como las organizaciones sociales, siguen teniendo un rol relevante de mediación en la sociedad y gran poder en la construcción de agenda.

Es importante señalar que las diez cuentas con más RT- identificadas en el apartado 4.1- se ubicaron en ocho comunidades distintas de la red, lo que significa una buena repartición dentro de ella.

Del grafo inferimos, también, que los módulos adoptan el arquetipo "comunidades agrupadas"; esto es, los/las usuarios/as se han agrupado cerca de cuatro grandes nodos que actuaron como fuentes informativas. Alrededor de esos centros se formaron las conversaciones. Son grupos con su propia audiencia, influencers y fuentes de información. Se trata de clusters que comparten un interés común por el proyecto de ley 018 pero con distintos enfoques.

\section{Conclusiones}

El estudio de la conversación digital en torno al \#RespetoAlDolorDeMadre y de sus características aporta algunas claves para entender lo que ha ocurrido con este hashtag en el contexto nacional. En principio, pues, resulta importante en un país como Panamá que cuenta con escasa producción académica acerca de su movimiento feminista y del uso que puede hacer de las plataformas digitales. Adicionalmente, es un aporte útil en la región centroamericana por las dificultades que conocen sus feminismos en términos de marginalización de su agenda.

El análisis empírico que hemos realizado sobre sobre la etiqueta nos permite sintetizar los hallazgos que se corresponden con los objetivos específicos de la investigación: 1) las cuentas más retuiteadas fueron las de las activistas feministas y académicos/as especialistas en la problemática; 2) las cuentas más mencionadas por los/las usuarios/ as pertenecen a tomadores de decisión (sistema político); 3) la conversación digital registra comunidades agrupadas con conexiones sólidas e interacciones densas (Smith, et al., 2014).

La discusión digital entorno a la etiqueta \#RespetoAlDolorDeMadre se convirtió en escenario de la oposición feminista al proyecto de ley 018 en Panamá, tal como había ocurrido en otros países con etiquetas como \#MeToo, \#NiUnaMenos o \#YoSíTeCreo. El impacto de la conversación digital fue tal que tuvo repercusiones en la agenda mediáticas y en la política; el proyecto no volvió a ser tratado por la Comisión de la Mujer durante el año 2019.

Es difícil concluir si el hashtag como tal y la conversación digital en torno a él contribuye de forma perenne a la causa feminista. La diversidad de personas involucradas en la discusión y los diferentes enfoques de las comunidades sobre el tema sugieren que el "éxito" puntual de la etiqueta no puede entenderse como un peldaño en la lucha 
feminista, tal como lo han sido otros como el \#MeToo y el \#NiUnaMenos que impactaron en la agenda pública global y produjeron cambios profundos (Acosta, 2018a; Laudano, 2017; Jaffe, 2018; Mendes et al., 2018; Rodino, 2018). Con todo, es probable que la sociedad civil panameña haya tomado mayor consciencia de las posibilidades ofrecidas por las redes sociales.

El trabajo actual no incluye el análisis de contenido de los tuits, por lo que una agenda de investigación futura podría incluir esta metodología a fin de determinar con mayor precisión el alcance de la conversación, eventualmente por comunidad, lo que podría confirmar o infirmar la hipótesis de que las distintas comunidades no actuaban según un solo interés. Nuestro análisis tampoco incluye del hashtag antagonista \#TuNombreMiRecuerdo, lo que igualmente podría constituir una agenda investigativa futura, con la dificultad de que ha sido una etiqueta utilizada de forma internacional, por lo que habría que aislar la conversación digital panameña.

Por el momento, podemos señalar que la plataforma Twitter permitió amplificar la voz de un movimiento feminista bastante reducido en el país, conectar con otras personas no necesariamente afines a él y visibilizar una temática privada (las pérdidas de embarazo y la violencia obstétrica que acompaña a menudo estos momentos), a través de expresiones masivamente compartidas. Queda aún, como advierte Loza (2014), que las activistas feministas puedan transformar los debates en la red en un verdadero movimiento social que trascienda las plataformas digitales.

\section{Bibliografía}

Aparicio, F. et Al. (2002): Historia de los movimientos de mujeres en Panamá en el Siglo XX. Panamá: Instituto de la Mujer de la Universidad de Panamá.

Abrego, V.H., Bona, Y. \& Reguillo, R. (2018): “Inteligencia artificial electoral. Tercer \#DebateINe a la Presidencia". En El efecto AMLO. Guadalajara: ITESO. Disponible en Internet: https://analisisplural.iteso.mx/2018/10/10/inteligencia-digital-electoral2018-tercer-debateine-a-la-presidencia/ [Consulta: Octubre de 2019].

Abernal (17 de septiembre de 2019): Corina Cano aclara que su proyecto no habla del aborto. Nex. Disponible en Internet: https://www.nexpanama.com/corina-canoaclara-que-su-proyecto-no-habla-del-aborto/

Acosta, M. (2018a): "Ciberactivismo feminista. La lucha de las mujeres por la despenalización del aborto en Argentina", en Sphera Publica, vol. 2, n 18, pp. 2-20.

Acosta, M. (2018b): “Violencia de género y tecnopolítica. La génesis del \#NiUnaMenos en Argentina". En: Salazar, R. y Zebadúa (ed.): Las formas de violencia en América Latina contemporánea. Buenos Aires: El Aleph.com, pp. 113-137.

Acosta, M., \& Lassi, A. (2019): “\#8M 2019. La conversación digital durante la Huelga Internacional de Mujeres", en Revista Arbitrada Interdisciplinaria Koinonía, vol. 5, nº 9, pp. 86-109. http://dx.doi.org/10.35381/r.k.v5i9.516

Acosta, M., \& Lassi, A. (2019): “Indignación online. La conversación digital del \#NiñasNoMadres en Argentina", en Comunicación y Medios, n 40, pp. 200-213. doi:10.5354/0719-1529.2019.53312

Alcazan, A.et al. (2012): Tecnopolítica, Internet y R-evoluciones. Barcelona: Icaria.

Alianza Panameña por la vida y la familia (2 de septiembre 2019): Triste que hay médicos que no les interesa que algunas mujeres no quieran que los restos de sus hijos [...] \#TuNombreMiRecuerdo. [Video adjunto]. Facebook. https://www.facebook.com/ AlianzaPanamena/videos/660722597744193/ 
Arce, M. (2008): “Panamá. Una deuda pendiente: la igualdad de oportunidades para la participación política de las mujeres". En: Llanos, B. y Sample, K. (eds.): Del dicho al hecho: Manual de buenas prácticas para la participación de mujeres en los partidos políticos latinoamericanos. Lima: Idea Internacional.

Arias Rodríguez, A. (2017): “La cimentación social del concepto mujer en la red social Facebook. Revista de Investigación Educativa", vol. 35, n 1, pp.181-195.

Arroyas-Langa, E., Martínez-Martínez, H. \& Berná-Sicilia, C. (2018): Twitter como espacio alternativo a la esfera política institucional. Análisis retórico de las estrategias discursivas de Podemos durante la moción de censura contra Rajoy. In J. Segarra, T. Hidalgo \& R. Rodríguez (Coords.). Actas de las Jornadas Científicas Internacionales sobre Análisis del discurso en un entorno transmedia (pp. 85-94). Alicante: Colección Mundo Digital de Revista Mediterránea de Comunicación. https://www.doi.org/10.14198/ medcom/2017/11cmd

Brown Araúz, H. (2020): “Elecciones Panamá 2019: un nuevo desvío”. En América Latina vota: elecciones 2017-2019. Madrid: Tecnos.

Bonilla, G. (2007): “La lucha de las mujeres en América Latina”, en Palobra, n 8, pp. 42-59.

Cammaerts, B., Mattoni, A. \& McCurdy, P. (Eds.) (2013): Mediation and protest movements. Bristol: Intellect.

Cammaerts, B. (2012): Protest logics and the mediation opportunity structure. European Journal Communication, 27 (2), 117-134.

Cano,C. [@cano_corina].(14 de marzo de 2019):Mi primer proyecto de Ley como Diputada del 8.7 es BLINDAR a Panamá de absolutamente todo lo que implica IDEOLOGIA DE GENERO!!! \#VotoProFamilia \#VotoFamilia2019 [post en Twitter]. Disponible en Internet:https://twitter.com/cano_corina/status/1106179947725504513 [Consulta: Octubre de 2019].

Carrasquilla Reina, M. A. (12 de septiembre de 2019): \#RespetoAlDolorDeMadre: la campaña en contra de la ley que daría identidad a nonatos. La Estrella de Panamá, nacionales. Disponible en Internet: https://www.laestrella.com.pa/nacional/190912/ ley-daria-campana-identidad-respetoaldolordemadre [Consulta: Noviembre de 2019].

Caro, L. (2015): "Construir y comunicar un "nosotras" feminista desde los medios sociales. Una reflexión acerca del "feminismo del hashtag", en Commons, vol. 4, n², pp. 124-154 https://revistas.uca.es/index.php/cayp/article/view/3098

Casero-Ripollés, A. (2015): “Estrategias y prácticas comunicativas del activismo político en las redes sociales en España", en Historia y Comunicación Social, vol. 20, n² 2, pp. 533-548.

Castelló, A. (2013): El uso de Twitter como canal de información y conversación por parte de los medios convencionales. Comunicación presentada en el XIV Foro de Investigación en Comunicación, Valencia, 18-19 febrero. Disponible en Internet: https://rua.ua.es/dspace/bitstream/10045/26898/1/Araceli_Castello_Martinez_1.pdf [Consulta: Noviembre de 2019].

Castells, M. (2009): Comunicación y poder. Madrid: Alianza.

Castells, M. (2012): Redes de indignación y esperanza. Madrid: Alianza.

Centro Internacional de Estudios Políticos y Sociales (En prensa): Encuesta de Ciudadanía y Derechos. Panamá: CIES.

Clark, R. (2016): "Hope in a Hashtag: The Discursive Activism of \#WhylStayed", en Feminist Media Studies, vol. 16, n 5, pp. 788-804. https://www.doi.org/10.1080/14680777.201 6.1138235 . 
Cohen, R. \& Rai, S. (2000) : Global Social Movements. New York: The Athlone Press.

Conferencia Episcopal Panameña (12 de septiembre de 2019): Comunicado de la Oficina de Comunicación de la CEP: El derecho al registro de identidad del niño en el vientre. Disponible en Internet: https://twitter.com/lglesiaPA/status/1172526832836628481 [Consulta: Enero de 2020].

Congosto, M. (2015): “Elecciones Europeas 2014: Viralidad de los mensajes en Twitter", REDES- Revista hispana para el análisis de redes sociales, vol. 26, $\mathrm{n}^{\circ}$ 1, pp. 23-52. DOI: 10.5565/rev/redes.529

Cook, J. \& Hasmath, R. (2014): The discursive construction and performance of gendered identity on social media, en Current Sociology, vol. 62, n 7, pp. 975-993. https://doi. org/10.1177/0011392114550008

Cruells, M. \& Ibarra, P. (Eds.) (2013): La democracia del futuro: Del $15 \mathrm{M}$ a la emergencia de una sociedad civil viva. Barcelona: Icaria.

Dankhe, G. L. (1986): “Investigación y comunicación”. En: Fernández Collado, C. y Danke, G. (eds.): La comunicación humana ciencia social. México: McGraw Hill, pp. 385-454

del Campo García, E. y Resina de la Fuente, J. (2010): “Redes sociales, ciberpolítica y nuevas movilizaciones: el impacto digital en los procesos de deliberación y participación ciudadana". Ponencia presentada en el X Congreso Español de Sociología, Universidad Pública de Navarra, Pamplona, España.

Día del Niño por Nacer, 25 de marzo. (sin fecha): Aciprensa. Disponible en Internet: https://www.aciprensa.com/vida/pornacer2.htm [Consulta: Octubre de 2019].

Diez, R. y Laraña, E. (2017): Democracia, dignidad y movimientos sociales. El surgimiento de la cultura cívica y la irrupción de los indignados en la vida pública. Colección Monografías, Núm. 308. Madrid: CIS.

Hossana Visión [@hosannavision]. (2 de abril de 2019) Hoy en tu programa La Verdad, nuestro presentador Roderick Burgos y su invitado Luis Ramírez, Psicólogo Clínico, nos conversarán sobre las Niñas Embarazadas. Hoy, 5:00pm por nuestra señal. [post en Twitter] Disponible en Internet: https://twitter.com/hosannavision/ status/1113128215642587141?s=20 [Consulta: Septiembre de 2019].

Fotopoulou, A. (2016): “Digital and networked by default? Women's organisations and the social imaginary of networked feminism", en New Media and Society, vol. 18, $\mathrm{n}^{\circ} 6$, pp 1-17. https://doi.org/10.1177/1461444814552264

Gago, R. (2019): Ciberfeminismo en España: discurso teórico y prácticas digitales. Alicante: Avicam Ediciones.

Garcés Évila, L. (21 de julio de 2019): “Quién critica el proyecto, no lo ha leído” dice la diputada Cano. El Siglo. Disponible en Internet: http://elsiglo.com.pa/panama/quiencritica-proyecto-leido-dice-diputada-cano/24130850 [Consulta: Octubre de 2019].

García, A. \& Silva, A. (2017): “Ciberfeminismo o feminismo en la Red”, en Antropología experimental, $\mathrm{n}^{\circ}$ 17, pp. 277-286. Disponible en Internet: https://revistaselectronicas. ujaen.es/index.php/rae/article/ view/3515 [Consulta: Octubre de 2019].

Gerbaudo, P. (2012): Tweets and the streets. Social media and contemporary activism. London: Pluto Press.

Gerbaudo, P. (2014): "The persistence of collectivity in digital protest. Information", en Communication \& Society, vol. 17, $\mathrm{n}^{\circ} 2$, pp. 264-268. 
Giraldo-Luque, S.; Fernández-García, N.; Pérez-Arce, J. C (2018): “La centralidad temática de la movilización \#NiUnaMenos en Twitter", en El profesional de la información, vol. 27, nº 1, pp. 96-105.

González, N. (8 de julio de 2016): Abogada cuestiona tema de ideología de género en iniciativa de educación sexual. Telemetro. Recuperado de: https://www.telemetro.com/ nacionales/2016/07/08/abogada-cuestiona-ideologia-iniciativa-educacion/1412564. html [Consulta: Octubre de 2019].

González, N. (14 de julio de 2016). "Queremos una ley hecha por los panameños" Cano sobre educación sexual. Telemetro. Disponible en: https://www.telemetro. com/nacionales/2016/07/14/queremos-hecha-panamenoscano-educacionsexual/1410041.html

Gupta, S. \& Kim, H.-W. (2004): "Virtual community: Concepts, implications, and future research directions". In Procs of the 10th Americas conf on information systems, pp. 2679-2687. Disponible en Internet: http://www.virtual-communities.net/mediawiki/ images/4/4a/SIGEBZ05-1115.pdf [Consulta: Septiembre de 2019].

Haro, C. y Sampedro, V. (2011): "Activismo político en Red: del Movimiento por la Vivienda Digna al 15M", en Teknokultura, vol. 8, n², pp. 167-185.

Jaffe, S. (2018) : "The Collective Power of \#MeToo", en Dissent, vol. 65, n² 2, pp. 80-87. doi:10.1353/dss.2018.0031.

Jiménez, C. (2016): “Modelo de análisis y monitoreo de hashtags de Twitter en campañas político-electorales". En Premio nacional de investigación social y de opinión pública 2016. México: Cámara de Diputados.

Larrondo, A ; Morales i Gras, J. \& Orbegozo Terradillos, J. (2019): "Feminist hashtag activism in Spain: measuring the degree of politicisation of online discourse on \#YoSíTeCreo, \#HermanaYoSíTeCreo, \#Cuéntalo y \#NoEstásSola", en Communication \& Society, vol. 32, n 4, pp. 207-221.

Latinobarómetro (2018): Datos de encuesta de Panamá. Disponible en Internet: http:// www.latinobarometro.org/lat.jsp

Laudano, C. (2017): Movilizaciones \#NiUnaMenos y \#VivasNosQueremos en Argentina. Entre el activismo digital y el \#FeminismoLoHizo. Seminário Internacional Fazendo Gênero 11 \& 13th Women's Worlds Congress (Anais Eletrônicos), Florianópolis. Disponible en Internet: http://www.wwc2017.eventos.dype.com.br/resources/ anais/1503871106_ARQUIVO_Laudano_Texto_completo_MM_FG.pdf [Consulta: Septiembre de 2019].

Lazarsfeld, P. \& Merton, R. (1954): “Friendship as a Social Process: A Substantive and Methodological Analysis". In Freedom and Control in Modern Society. New York: Van Nostrand.

Loza, S. (2014): "Hashtag Feminism, \#SolidaritylsForWhiteWomen, and the Other \#FemFuture", en Ada: A Journal of Gender, New Media, and Technology, 5. Disponible en Internet: http://adanewmedia.org/2014/07/issue5-loza [Consulta: Septiembre de 2019].

Marco, Y. (1997a): “El feminismo de los años veinte y la redefinición de la femeneidad en Panamá". En E. Rodríguez (ed.): Entre silencios y Voces. Género e Historia en América Central (1730-1990). San José: Editorial Universidad de Costa Rica.

Marco, Y. (1997b): “El nacimiento del movimiento feminista en Panamá, 1923". En: Ciudadanas. Una Memoria Inconstante. Caracas: Nueva Sociedad, CDE Centro de Cocumentación y Estudios. 
Marco, Y. (2002): "Ser ciudadana en Panamá en la década de 1930". En Un siglo de luchas femeninas en América Latina. San José: Editorial Universidad de Costa Rica.

Marco, Y. (2005): “Ruptura de la tradición, construcción de la mujer moderna y resistencia del patriarcado liberal en Panamá en las décadas de 1920 y 1930", en Diálogos: Revista electronica de historia, vol. 5. Disponible en Internet: https://revistas.ucr.ac.cr/index. php/dialogos/article/view/6247/5950 [Consulta: Septiembre de 2019].

Marco, Y. (2007): Clara González de Behringer: Biografía. Panamá: Edición Hans Roeder.

Marco, Y. (2010): “Tras las huellas de la utopia sufragista. Las luchas por el sufragio femenino en Panamá, 1923-1946", en Procesos Sociales, n² 2, pp. 115-136.

Marco, Y. (2018): "Los debates acerca de la condición femenina y el feminismo en Panamá. 1911-1922", en Revista del CESLA, n²1, pp. 89-104.

Mendes, K., Ringrose, J. \& Keller, J. (2018): “\#MeToo and the promise and pitfalls of challenging rape culture through digital feminist activism", en European Journal of Women's Studies, vol. 25, n² 2, 236-246. https://doi.org/10.1177/1350506818765318

Molpeceres Arnaiz, S. y Filardo Llamas, S. (2020): Llamamientos feministas en Twitter: ideología, identidad colectiva y reenmarcado de símbolos en la huelga del 8M y la manifestación contra la sentencia de 'La Manada', en Dígitos. Revista de Comunicación Digital, n6, pp. 55-78.

Moreno de Cuvillier, L. (2017): “Movilización social de las mujeres en Panamá". Societas, vol. 19, n², pp. 61-74.

Nevache, C. (2017): Iglesias evangélicas en Panamá: análisis de la emergencia de un nuevo actor político, en Revista Panameña de Política, n²4, pp. 63-101.

Nevache, C. (2018): “Panamá: Evangélicos ¿del Grupo de Presión al Actor Electoral?”. En: Pérez, J. y Grundberger, S. (eds.): Evangélicos y poder en América Latina. Lima: Instituto de Estudios Social Cristianos y Konrad Adenauer Stiftung, pp. 377-404.

Núñez Puente, S. \& Fernández Romero, D. (2017): Narrativas transformadoras y testimonio ético: las estrategias discursivas de la Plataforma Feminista 7N, Contra las Violencias Machistas. index.comunicación, vol. 7, , n³ 3, pp. 269-281. Disponible en Internet: http://journals.sfu.ca/indexcomunicacion/index.php/indexcomunicacion/ article/view/31 [Consulta: Septiembre de 2019].

Orbegozo Terradillos, J., Morales i Grass, J. y Larrondo Ureta, A. (2019): "Feminismos indignados ante la justicia: la conversación digital en el caso de La Manada", en IC: Revista Científica de Información y Comunicación, , n 16, pp. 211-247. https://dx.doi. org/10.12795/IC.2019.i01.08

Piñuel, J. L. (2002): “Epistemología, metodología y técnicas del análisis de contenido”, en Sociolinguistic Studies, vol. 3, n 1, pp. 1-42. Disponible en Internet: https://www.ucm. es/data/cont/docs/268-2013-07-29-[Consulta: Septiembre de 2019].

Pontificium opus a sancta infantia (1999). Día del niño por nacer. Disponible en Internet: http://www.vatican.va/roman_curia/congregations/cevang/p_missionary_works/ infantia/documents/rc_ic_infantia_doc_20090324_boletin7p14_sp.html

Proyecto de ley 018. De Identidad para Bebés fallecidos en el vientre materno. Asamblea Nacional de Panamá, 15 de julio de 2019. Recuperado de: https://asamblea.gob.pa/ APPS/SEG_LEGIS/PDF_SEG/PDF_SEG_2010/PDF_SEG_2019/2019_A_029.pdf

Reig, D. (2012): “Disonancia cognitiva y apropiación de las TIC”, en TELOS. Cuadernos de comunicación e innovación, vol. 90, nº 1, pp. 9-10. 
Resina de la Fuente, J. (2010): "Ciberpolítica, redes sociales y nuevas movilizaciones en España: el impacto digital en los procesos de deliberación y participación ciudadana", en Mediaciones Sociales, $\mathrm{n}^{\circ}$ 7, pp.143-164.

Rodino-Colocino, M. (2018). "Me too, \#MeToo: countering cruelty with empathy", en Communication and Critical/Cultural Studies, vol. 15, n 1, pp. 96-100. https://doi.org/1 $0.1080 / 14791420.2018 .1435083$

Sádaba, I. (2012): "Acción colectiva y movimientos sociales en las redes digitales. Aspectos históricos y digitales", en ARBOR. Ciencia, Pensamiento y Cultura, vol. 188, n 756, pp. 781-794.

Sádaba, I. y Barranquero, A. (2019): "Las redes sociales del ciberfeminismo en España: identidad y repertorios de acción", en Athenea Digital, vol. 19, n 1, e2058. https://doi. org/10.5565/rev/athenea.2058

Sampedro, V. (2005): 2005. 13 M multitudes on line. Madrid: Los Libros de la Catarata.

Sampedro, V. y Resina de la Fuente, J. (2010): “Opinión pública y democracia deliberativa en la Sociedad Red", en Revista Ayer, $n^{\circ}$ 4, pp. 139-162.

Santini, R.M, Terra, C. y Duarte de AlmeAda, A. (2017): Feminismo 2.0: A mobilização das mulheres no Brasil contra o assédio sexual através das mídias sociais (\#primeiroassedio). P2P e inov, vol. 3, no 1, pp. 148-168. https://doi.org/10.21721/ p2p.2016v3n1.p148-164

Smith, M. A., Rainie, L., Shneiderman, B., \& Himelboim, I. (2014) : Mapping Twitter topic networks: From polarized crowds to community clusters. Pew Research Center, 20, 1-56. Recuperado de: https://www.pewinternet.org/2014/02/20/mapping-twittertopic-networks-from-polarized-crowds-to-community-clusters/

Sorj, B. y Fausto, S. (Comps.) (2016): Activismo político en tiempos de Internet. San Pablo: Plataforma Democrática.

Szwako, J. L. y Sivori, H. F. (2019): "Not about religion." Institutional discourses and interactions in the presidential indictments against F. Lugo in Paraguay (2012) and D. Rousseff in Brazil (2016). Ponencia presentada en el Congreso Feminismos y Conservadurismos en América Latina. Ciudad de México.

Tarullo, R. y García, M. (2020): “Hashtivismo feminista en Instagram: \#NiñasNoMadres de @actrices.argentinas", en Dígitos. Revista de Comunicación Digital, n6, pp.31-54.

Treré, E. y Barranquero, A. (2013): “De mitos y sublimes digitales: movimientos sociales y tecnologías de la comunicación desde una perspectiva histórica", en Redes.com, $\mathrm{n}^{\circ}$ 8 , pp. 27-47.

Ungo, U. (2000): Para cambiar la vida: política y pensamiento del feminismo en América Latina. Panamá: Instituto de la Mujer.

Verea, C. P. (2004): "Malas madres": la construcción social de la maternidad. Debate feminista, vol. 30, n 15, pp. 12-34.

Welp, Y. (2015): Cuando todo lo sólido se desvanece en Twitter. Análisis del Movimiento Social \#YOSOY132 (México 2012), en Posdata, vol. 20, n² 2, pp. 417-439.

Zafarani R.; Ali Abbasi, M.; Liu, H. (2014): Social Media Mining: an Introduction. Cambridge: Cambridge University Press. http://dx.doi.org/10.1017/cbo9781139088510 